\title{
Competencies for structured professional development of neonatal nurses
}

\section{in South Africa}

\author{
C Maree, ${ }^{1}$ BCur, BA Cur, MCur, PhD, PGCHE; M Scheepers, ${ }^{2}$ BCur, MCur, PhD; E S Janse van Rensburg, ${ }^{3}$ BCur, MCur, DCur \\ ${ }^{1}$ Department of Nursing Science, Faculty of Health Sciences, University of Pretoria, South Africa \\ ${ }^{2}$ Life College of Learning, Life Healthcare, Illovo, Johannesburg, South Africa \\ ${ }^{3}$ Department of Health Studies, School of Social Sciences, College of Human Sciences, University of South Africa, Pretoria, South Africa
}

Corresponding author: C Maree (carin.maree@up.ac.za)

\begin{abstract}
Background. The unpredictability of any illness at birth, and recovery from such an illness after birth, create challenges for nurses involved in neonatal care, and require competent nurses in the period following birth in both resource-limited and technologically advanced contexts. Neonatal nursing emerged worldwide as a nursing specialty over the last five decades to meet these challenges through in-depth knowledge of healthy, preterm and ill neonates. There is a high demand for neonatal nurses to lower neonatal mortality and morbidity, negative media exposure and litigation.

Objectives. To reflect on a competency framework for nurses in neonatal practice to enhance professional development that is context specific.

Methods. A competency framework for nurses involved in neonatal practice was developed and validated through nominal group techniques, literature control and Delphi techniques.

Results. Although nurse training is done at tertiary education institutions, there is a gap in ensuring consistencies in clinical performance and professional development from novice to expert, especially in a field such as neonatal care. Professional development can enhance the standard of neonatal care, especially if linked to competencies that are specific to the neonatal context.

Conclusion. A competency framework has an important role to play in equipping nurses in neonatal practice with the knowledge and skills required to reduce the persistent neonatal mortality and morbidity rates in South Africa.
\end{abstract}

Afr J Health Professions Educ 2020;12(3):154-160. https://doi.org/10.7196/AJHPE.2020.v12i3.1364

Competent nursing staff in the neonatal intensive care unit (NICU) can make the difference between life and death for sick and premature babies. Unique challenges exist in neonatal practice owing to a high-risk period of adaptation in the days after birth, whether the context is resource limited or equipped with the newest technology. Globally, in 2016, 2.7 million babies died in their first month of life, and an additional 2.6 million were stillborn. ${ }^{[1]}$ Fifteen million babies ( 1 in 10 live births) are born prematurely every year, one million of whom die due to complications of prematurity. ${ }^{[2]}$ Neonatal mortality is a serious problem worldwide.

Newborn infants who need special care are prone to suffer injury, with long-term morbidity or even death. Neonatal outcomes can only be optimised and sustained if the nurses who are responsible for the care have the necessary knowledge and skills. ${ }^{[3]}$ It is, however, important to clarify exactly what the specific knowledge, skills and attributes (competencies) should be. The working group of the Council of International Neonatal Nurses $^{[4]}$ has stated that 'neonatal nursing is a highly specialised field. To meet the complex needs of neonates and their families, there must be uniform qualifications and competencies to support standard knowledge and clinical performance.'

In the South African (SA) context, nurses play a key role in neonatal practice, but they have various levels of competency as they are trained via different programmes with different levels of knowledge, skills and attributes. The SA Nursing Council (SANC) has not recognised neonatal nursing as an individual specialised qualification since $2012,{ }^{[5]}$ which results in a lack of training opportunities that focus on caring for ill and prematurely born infants, and specialised NICU care. Shift leaders play a crucial role in the management of the NICU and ensuring a high quality of care by neonatal nurses who have updated knowledge, skills and attributes. In the current situation in SA, the competency framework for professional development of nurses in neonatal practice can be used as an alternative to formal neonatal training, which may improve quality of care. The shift leader can utilise this competency framework for supervision and performance management, while professional, enrolled and auxiliary nurses can use it as a guide for practice.

A competency framework refers to standardised and integrated knowledge, skills and attributes, offering a structured description of competencies with the purpose of facilitating an understanding of the requirements of staff in a specific context. In the context of this study, the focus is on nurse specialists/ shift leaders, professional nurses and enrolled and auxiliary nurses in a variety of SA neonatal practice settings. ${ }^{[6]}$

Several neonatal competency frameworks have been developed over time. ${ }^{[7-11]}$ In developed countries, neonatal competencies seem to have comparable domains such as using research and evidence, leadership and professional development, communication, and legal and ethical considerations. ${ }^{[7,9-11]}$ None of these could be applied directly to the SA context, since neonatal care takes place in a variety of settings. These settings include the public and private sector and rural and urban settings. Neonatal care is provided by different categories of nurses, as neonatal nursing is 
no longer acknowledged as an individual specialised qualification by the SANC, but only as a component of midwifery and child nursing. ${ }^{[12]}$

The categories of nurses in neonatal practice are neonatal nurse specialists (trained between 2000 and 2012), nurses specialised in midwifery, critical care of child nursing, professional nurses acting as shift leaders (senior/ experienced professional nurses who take responsibility for decisions in neonatal practice) and professional nurses and enrolled nurses with a range of experience in neonatal care. Every category of nurses, enrolled or registered, has a scope of practice in SA. ${ }^{[13]}$ All nurses are bound to apply their specific knowledge and skills to provide safe, competent and ethical care, ${ }^{[14]}$ but there has not previously been a clear framework to inform what competencies each category of nurses should have in neonatal practice.

The competency framework developed in this study was, therefore, an attempt to clarify the needed competencies based on the generic competency framework of the SANC ${ }^{[15]}$ that could be applied for professional development in neonatal practice.

Professional development can be defined as 'the continuous process of acquiring new knowledge and skills that relate to one's profession, job responsibilities, or work environment. ${ }^{[16]}$ The competency framework for the professional development of different categories of nurses in neonatal practice was based on Benner's ${ }^{[17]}$ five stages of development to move from a novice to becoming an expert. Each individual nurse begins as a novice within his/her respective category when exposed to neonatal practice for the first time, and has the potential to move through the different levels towards expertise at his/her own pace, but not all will master all the levels.

Novice nurses are those beginners who have no experience of the situations in which they are supposed to perform. Rules are taught and applied generally in order to assist them with their performance. As they gain experience, they become advanced beginner nurses who can demonstrate marginally acceptable performance in real situations by applying formulated principles based on their experiences. ${ }^{[17]}$

Competent nurses develop when they observe their own actions in terms of the long-range goals they are consciously aware of, and formulate a plan based on abstract and analytical considerations of a problem. However, they do not have enough experience to recognise a situation in terms of an overall picture, or understand which aspects are most important. They become proficient nurses when they are able to observe or perceive a situation as a whole and not only in a separated part, and can identify when the normal does not realise and decide on appropriate actions. They become expert nurses when they have an immense background of knowledge and experience and can intuitively grasp a situation. They no longer rely on analysis to obtain an understanding of a situation before appropriate action is taken, and they work from a profound comprehension of the whole situation. ${ }^{[17]}$ The higher the level of professional development of nurses, the higher the expectation is that they will be able to deliver high-quality competent care to a specialised group of patients in the NICU within their specific range of responsibilities. Only proficient and expert professional nurses should be responsible for shift leading.

The focus of this study was therefore to provide a framework for structuring professional development to achieve competency in the different categories of nurses involved in neonatal care. This article, however, only reports on the competency framework for nurse specialists/shift leaders in neonatal practice, while the others are available on request.

\section{Methods}

A multi-method research design that applies two or more research methods in one project ${ }^{[18]}$ was used in the study in the form of consensus research. The types of consensus research methods included the nominal group technique, the Delphi method ${ }^{[19]}$ and a literature review in three phases. Ethical approval was obtained by the University of Pretorial Faculty of Health Sciences' Ethics Committee (ref. no. 93/2012).

\section{Phase 1}

In phase 1, consensus was reached on the competencies and their priorities needed by nurses in neonatal nursing practice. Seven nominal group discussions were held in SA in Gauteng, North West and Mpumalanga Provinces with registered nurses working in NICUs of private and public hospitals, 5 facilitated in the private sector and 2 in a public hospital setting. The number of participants varied between 4 and 7 members per nominal group, with a total of 32 participants to address the competencies of neonatal nurses.

\section{Phase 2}

During phase 2, a literature review was conducted on existing neonatal competency frameworks, and those included were the Royal College of Nursing (UK), ${ }^{[7]}$ Rwandan Competency Framework, ${ }^{[8]}$ Scottish Neonatal Nurses' Group, ${ }^{[9]}$ Australian College of Neonatal Nurses ${ }^{[10]}$ and the PanLondon Band 5 Competency Document. ${ }^{[1]}$ Through a process of inductive and deductive reasoning, the competencies were formulated as an abridged list of competencies and verified by seven neonatal nursing experts through the Delphi technique.

\section{Phase 3}

In phase 3, competency frameworks for professional development of nurses in neonatal practice were further unpacked based on the findings from phase 2, and aligned with the SANC's competency framework ${ }^{[15]}$ to make them applicable for the SA context. The competencies were verified with a literature control, and interpreted within Benner's levels from novice to expert, ${ }^{[20]}$ so that the framework can be used for professional development in nursing practice. The competency framework of the neonatal specialists/ shift leaders was formulated first, and thereafter adjusted for the enrolled nurses and auxiliary nurses based on their respective scopes of practice. The competency frameworks were validated by an expert panel consisting of six experts in the field of neonatal nursing in the SA context, using the Delphi technique.

\section{Results}

Neonatal nurses serve a unique and vulnerable population for which they need specific competencies. The competencies are divided according to professional, ethical and legal practice; care provision and care management; and personal development and quality of care to be aligned with the SANC competency framework. ${ }^{[15]}$ The competencies for the neonatal specialists/shift leaders as derived during the different phases of this study are illustrated in Tables 1, 2 and 3, and should be interpreted within Benner's framework ${ }^{[17,20]}$ (last columns of tables) for self-evaluation or for performance management purposes. 
Table 1. Competencies of neonatal nurse specialists/shift leaders related to professional, ethical and legal practice

\begin{tabular}{|c|c|c|c|c|c|}
\hline \multirow[b]{2}{*}{ Competency } & \multicolumn{5}{|c|}{ Benner's levels of professional development (to be completed by user) } \\
\hline & Novice & Advanced beginner & Proficient & Competent & Expert \\
\hline \multicolumn{6}{|l|}{ A1: Professional practice } \\
\hline \multicolumn{6}{|l|}{$\begin{array}{l}\text { Display professional autonomy, accountability and responsibility within scope } \\
\text { of practice }{ }^{[13]}\end{array}$} \\
\hline \multicolumn{6}{|l|}{ Practise reflectively and in evidence-based manner ${ }^{[21]}$} \\
\hline \multicolumn{6}{|l|}{ Act as a role model and leader ${ }^{[21]}$} \\
\hline \multicolumn{6}{|l|}{ Function as part of, or co-ordinate, an interprofessional healthcare team ${ }^{[2]}$} \\
\hline \multicolumn{6}{|l|}{ A2: Ethical practice } \\
\hline \multicolumn{6}{|l|}{$\begin{array}{l}\text { Adhere to ethical principles and bioethical decision-making in practice, and } \\
\text { facilitate ethical awareness e.g. through presenting workshops }{ }^{[23]}\end{array}$} \\
\hline \multicolumn{6}{|l|}{ Protect human rights and advocate for neonates and their families ${ }^{[24]}$} \\
\hline \multicolumn{6}{|l|}{$\begin{array}{l}\text { Facilitate sensitivity to cultural, religious, language, professional and technological } \\
\text { diversity in neonatal care }{ }^{[25]}\end{array}$} \\
\hline \multicolumn{6}{|l|}{ A3: Legal practice } \\
\hline $\begin{array}{l}\text { Ensure compliance with relevant acts, regulations, policies, guidelines, protocols } \\
\text { and algorithms }{ }^{[13,21,23]}\end{array}$ & & & & & \\
\hline
\end{tabular}

\section{Professional, ethical and legal practice}

In the SANC competency framework, ${ }^{[15]}$ accountability and the responsibility for one's own judgement, outcomes of care and continued competence according to legislation is noted, and ethical practice is described in terms of the nurses' code of conduct, ethical decision-making, advocacy and professional integrity. The related competencies in neonatal nursing practice are captured in Table 1.

Neonatal specialists/shift leaders should be able to display professional autonomy, accountability and responsibility within their scope of practice. ${ }^{[13]}$ They need to practise reflectively and in an evidence-based manner, while they act as a role model and leader in neonatal practice. ${ }^{[21]}$ They are also expected to function as part of, or to co-ordinate, an interprofessional healthcare team in the NICU to the benefit of patients and families. ${ }^{[22]}$

A crucial requirement for neonatal specialists/shift leaders is adherence to ethical principles, bioethical decision-making in practice, and facilitation of ethical awareness. ${ }^{[23]}$ They have the responsibility to protect human rights and to advocate for neonates and their families ${ }^{[24]}$ and facilitate sensitivity to cultural, religious, language, professional and technological diversity in neonatal care. ${ }^{[25]}$

In terms of legal practice, they need to ensure compliance with relevant acts, regulations, policies, guidelines, protocols and algorithms. ${ }^{[13,21,23]}$

\section{Care provision and care management}

Care provision and care management refers to the underpinning of the provision of care including health promotion, assessment, diagnosis, planning and implementation, evaluation, recording and information management, and therapeutic communication and relationships. ${ }^{[10]}$ The related competencies for neonatal specialists/shift leaders are captured in Table 2 .

In terms of health promotion, neonatal nurse specialists/shift leaders should be able to create awareness, and provide care and health education. They need to understand the continuum of care and the need to advocate for improved family planning, maternal health, antenatal and intrapartum care in order to improve neonatal outcomes. ${ }^{[26]}$ This implies the application of the Essential Care of Every Baby ${ }^{[27]}$ guidelines for neonates with common illnesses, prevention of disability of very preterm and critically ill neonates, hygiene and infection prevention, ${ }^{[29]}$ developmental care (including skin-toskin care), ${ }^{[28]}$ exclusive breastfeeding ${ }^{[31]}$ and immunisation. ${ }^{[32]}$

The core of their responsibilities lies with assessment, diagnosis, planning and implementation of care of preterm, very preterm, ill and critically ill neonates. To be competent, they need to have specific prior knowledge, be able to assess, diagnose, plan and implement neonatal care, be skillful in resuscitation and transport of neonates and demonstrate technical competence in the NICU. ${ }^{[33-37]}$

The prior knowledge that they should possess should enable competency in neonatal care related to: the principles and application of the nursing process in neonatal practice, and creating a safe, secure and therapeutic environment for these neonates; fetal and neonatal development, anatomy, physiology, pathophysiology and genetic disorders affecting neonates; the principles and application of neonatal pharmacology and medication management; and the principles and application of developmental care, including the 'golden hour', skin-to-skin care, kangaroo care and facilitation of bonding and attachment. ${ }^{[33-37]}$

They should be able to assess and diagnose conditions related to the different systems of any neonate using history taking, physical assessment, vital data assessment and diagnostic investigations, ${ }^{[33-37]}$ including but not limited to aspects mentioned in Table 2. They are required to conduct basic and advanced resuscitation of neonates. ${ }^{[35]}$

Neonatal nurse specialists/shift leaders are expected to plan and implement care of the preterm, very preterm, ill and critically ill neonate by means of application of the principles of Essential Care of Small Babies, ${ }^{[35]}$ advanced medical and surgical neonatal nursing care, palliative care and medication management. The application is related to the respiratory, cardiovascular, neurological, endocrine, musculoskeletal, genitourinary, gastrointestinal, haematological and integumentary systems, eyes, ears, pain and complex multi-organ disorders. ${ }^{[33-37]}$ Related aspects to each of these are included in but not limited to those indicated in Table 2 .

They should be skilful in transport of the preterm, very preterm, ill and critically ill neonates (including skin-to-skin transport) internally in institutions, or by means of ambulance or being airlifted. ${ }^{[38]}$ Furthermore, 


\section{Research}

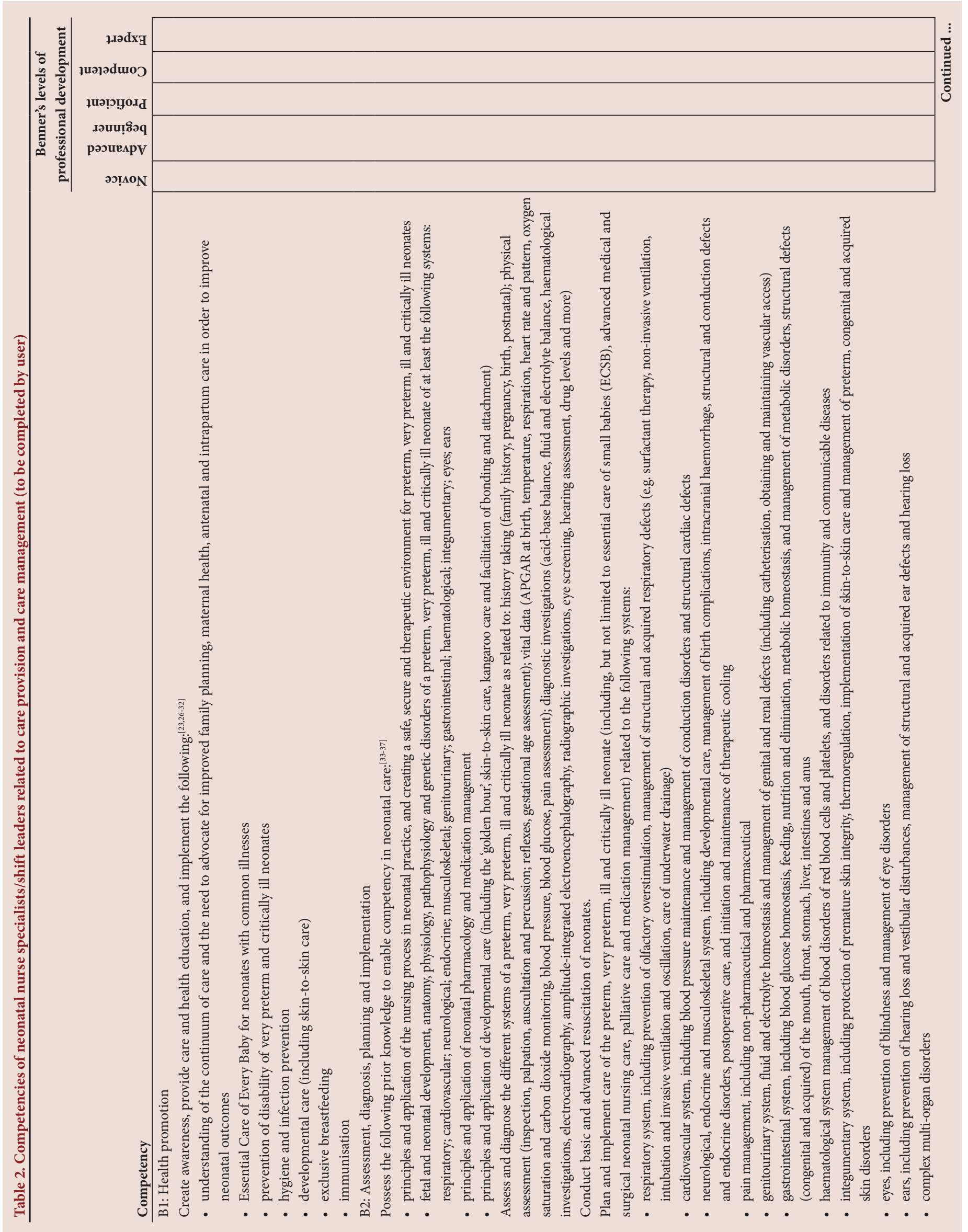




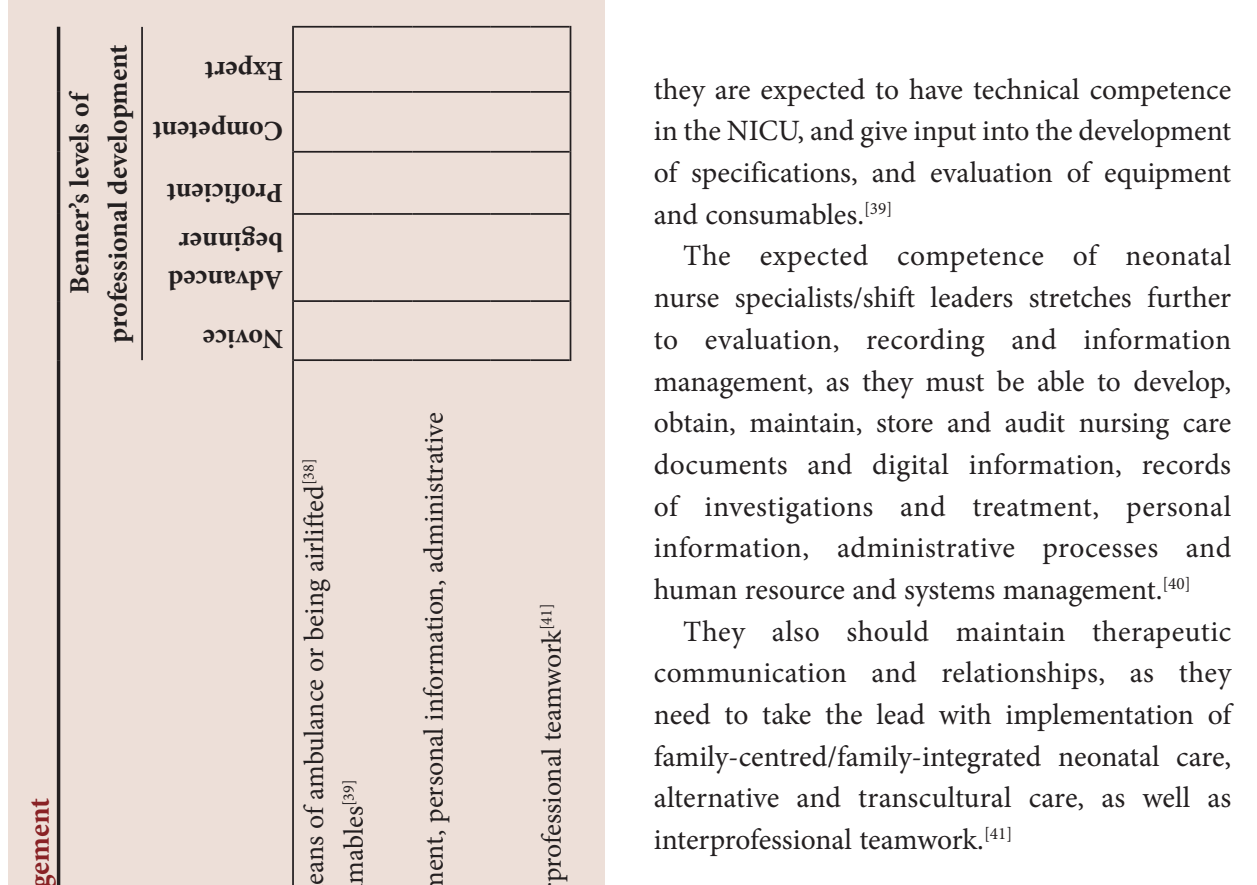

\section{Personal development and quality of} care

Lifelong learning enhances personal development. Neonatal specialists/shift leaders should adopt the culture of continuous learning to ensure that their competencies are updated and relevant. ${ }^{[46]}$ Specialised knowledge and skills is a requirement to ensure safe caring practices for neonates. ${ }^{[42-44]}$ The quality of care as reflected in neonatal mortality and morbidity can be enhanced by the design and implementation of quality improvement processes. Evidence-based practice ensures that research is translated into practice to enhance the quality of neonatal care. ${ }^{[43,44]}$ The management of human and material resources and staff development forms additional aspects of quality improvement, as indicated in Table 3.

The competencies for the neonatal nurse/ shift leader are addressed in the discussion of the results, and indicated in Tables 1 - 3 . The competencies for professional, enrolled and auxiliary nurses were derived from these, and adjusted according to their respective scopes of practice. ${ }^{[46]}$ The detailed competency frameworks for these categories are available on request.

\section{Discussion}

Neonates are vulnerable at birth and during the days following birth. ${ }^{[47,48]}$ Some neonates need to be hospitalised owing to illness and/or prematurity, and they need competent staff to take care of them. ${ }^{[23]}$ Unfortunately, inadequate care of these neonates and a lack of competent neonatal nurses pose a risk to quality and competent care for neonates. ${ }^{[47]}$
One option to improve the competency of staff working in neonatal practice is to focus on the professional development and performance management of individuals. The purpose of this study was to develop a competency framework that could be used as a tool to structure professional development and performance management of the different categories of nurse working in neonatal practice. Professional development implies advancement from one level to the next, from novice to expert, ${ }^{[17,20]}$ to meet the expected competencies. It is furthermore vital that nurses work within their scope of practice to ensure competent and quality care. ${ }^{[47]}$ In the SA context, the scope of practice of the different categories of nurse is prescribed by the SANC. ${ }^{[46]}$

With the expected competencies recognised, individual plans can be developed for professional development, and nurses can be acknowledged by suitable rewards and recognition, or merely by acknowledgement in work performance reviews. The potential value thereof is described by $\mathrm{Half}^{[49]}$ as a win for the entire team. One can increase the collective knowledge of the team by investing in the staff; as they become more effective, they become more confident and happier, and their job satisfaction also increases. The company becomes more appealing as an employer of choice, which, in turn, strengthens the staff retention strategy. ${ }^{[49]}$

The specific competencies needed in the NICU that can be strengthened through a competency framework and professional development include those related to professional, ethical and legal practice to enhance accountability and responsibility. ${ }^{[13,21-25]}$ Competencies should be established for the provision of care of preterm, very preterm, ill and critically ill neonates. Competencies are based on health promotion, assessment, diagnosing, planning, implementation, evaluation, recording and information management, therapeutic communication and interprofessional teamwork. ${ }^{[23,26-41]}$

Personal and professional development and performance management might contribute to lifelong learning, which, in turn, is expected to lead to continuous improvement of competency and work satisfaction. ${ }^{[49]}$ Furthermore, using a competency framework for professional development and performance management is expected to limit or reduce instances of injury to neonatal patients, and improve the outcomes for neonates and their families. ${ }^{[50]}$

The competency framework enhances practice by providing clear guidance on what 
Table 3. Competencies of neonatal nurse specialists/shift leaders related to personal development and quality of care

\begin{tabular}{|c|c|c|c|c|c|}
\hline \multirow[b]{2}{*}{ Competency } & \multicolumn{5}{|c|}{ Benner's levels of professional development (to be completed by user) } \\
\hline & Novice & $\begin{array}{l}\text { Advanced } \\
\text { beginner }\end{array}$ & Proficient & Competent & Expert \\
\hline \multicolumn{6}{|l|}{ C1: Quality improvement } \\
\hline \multicolumn{6}{|l|}{$\begin{array}{l}\text { Assess, plan, implement and evaluate quality improvement processes and initiatives } \\
\text { to address neonatal mortality and morbidity }{ }^{[42]}\end{array}$} \\
\hline \multicolumn{6}{|l|}{$\begin{array}{l}\text { Translate research findings into practice to contribute to setting standards and } \\
\text { development of best practice guidelines and policies }{ }^{[3,44]}\end{array}$} \\
\hline \multicolumn{6}{|l|}{ Manage human and material resources, including but not limited to budget, } \\
\hline \multicolumn{6}{|l|}{ Contribute to staff development and nursing practice through education ${ }^{[44]}$} \\
\hline \multicolumn{6}{|l|}{ C2: Continuing personal development } \\
\hline $\begin{array}{l}\text { Engage in a culture of lifelong learning and continuous professional development } \\
\text { and active involvement in a professional association }{ }^{[45]}\end{array}$ & & & & & \\
\hline
\end{tabular}

is expected of the nurse in neonatal practice. Education can be improved through professional development and in-service training of neonatal nurses according to the expected competencies. It is recommended that additional research be conducted on the implementation of the competency framework and the effect thereof on neonatal mortality and morbidity, and its contribution to the third sustainable development goal. ${ }^{[26]}$

\section{Study limitations}

The competency framework is developed for the SA context, but needs to be exposed to more participants for input before it can be generalised and adapted for other countries.

\section{Conclusion}

In order to deal with the vulnerability of neonates in the days following birth, the inadequate care of these neonates and the lack of competent neonatal nurses, a competency framework was developed. The competency framework is an attempt to assist with the challenges that pose a risk to quality and competent care of neonates, and provides a guide for training, supervision, delegation and performance management in the NICU.

Declaration. This study was completed as a requirement for a $\mathrm{PhD}$ qualification. Acknowledgments. The authors would like to thank SANTRUST for the funding received for the $\mathrm{PhD}$ study.

Author contributions. Planning of the document: MS with input from CM and EJvR. Data collection and analysis: MS under supervision of CM and EJvR. Manuscript writing: MS, CM and EJvR. Critical revisions for important intellectual content: MS, CM and EJvR.

Funding. This research received a funding grant from SANTRUST.

Conflicts of interest. None.

1. Liu L, Oza S, Hogan D, et al. Global, regional, and national causes of under-5 mortality in 2000-15: An updated systematic analysis with implications for the Sustainable Development Goals. Lancet 2016;388(10063):30273035. https://doi.org/10.1016/s0140-6736(16)31593-8

2. Chopra M, Daviaud E, Pattinson R, et al. Saving the lives of South Africas's mothers, babies, and children: Can the health system deliver? Lancet 2009;374(9692):835-846. https://doi.org/10.1016/s0140-6736(09)61123-5

3. Rhoda N, Kauchali S, Velaphi S, Gebhardt SGS, Barron P. Reducing neonatal deaths in South Africa: Progress and challenges. S Afr Med J 2018;108(3a):S9-S16. https://doi.org/10.7196/SAMJ.2017.v108i3b.12804

4. Council of International Neonatal Nurses. Position papers. COINN, 2020. http://coinnurses.org/wp-content uploads/2013/06/COINN-pos-paper.pdf (accessed 3 March 2020).

5. South African Nursing Council. Professional practice for nurses and midwives. Pretoria: SANC, 2020. https:// www.sanc.co.za/professional_practice.htm (accessed 3 March 2020).
6. Scheepers M. Development of a competency framework for the professional development of different categories of nurses in neonatal practice. $\mathrm{PhD}$ thesis. Pretoria: University of Pretoria, 2020.

7. Royal College of Nursing. Competence, education and careers in neonatal nursing: RCN guidance. London Royal College of Nursing, 2011. https://www.rcn.org.uk/professional-development/publications/pub-00464 (accessed 12 November 2019)

8. Ntigurirwa $\mathrm{P}$, Mellor K, Langer D, et al. A health partnership to reduce neonatal mortality in four hospitals in Rwanda. Globalization Health 2017:13(28). https://doi.org/10.1186/s12992-017-0252-6.

9. Scottish Neonatal Nurses' Group. The Competency Framework and Core Clinical Skills for Neonatal Nurses. SNNG, 2010. http://www.snng.org.uk/publications/datafiles/Career\%20Framework\%20for\%20Neonatal\%20 Support\%20Workers.pdf (accessed 12 November 2019).

10. Australian College of Neonatal Nurses. Competency standards for neonatal nurses. ACNN, 2012. http://www. acnn.org.au/resources/australian-standards-for-neonatal-nurses/ACNN-Standards-for-Neonatal-Nurses-2012. pdf (accessed 8 November 2019)

11. London Neonatal Network. Pan-London band 5 competency document. London: London Neonatal Network 2014. http://www.londonneonatalnetwork.org.uk/wp-content/uploads/2015/07/Pan-London-Neonatal-ODN Band-5-neonatal-competency-Document-2014-15-00000003.pdf (accessed 8 November 2019).

12. South Africa. Guidelines for maternity care in South Africa. A manual for clinics, community centres and district hospitals. 4th ed. Pretoria: National Department of Health, 2015. https://www.medbox.org/countries/guidelinesfor-maternity-care-in-south-africa/preview (accessed 3 March 2020).

13. Geyer N. A new approach to professional practice. Claremont: Juta, 2013:43.

14. South Africa. Nursing Act No. 33 of 2005.

15. South African Nursing Council. Generic competency framework for advanced practitioners. Pretoria Government Printers, 2014.

16. Oyetunde MO, Oluwafunke KI. Professional development and career pathway in nursing. Int J Sci 2015;4(6):92-104

17. Benner P. From novice to expert - excellence and power in clinical nursing practice. California: Addison-Wesley Publishing Company, 1984:13.

18. Cooper SJ, Kinsman L, Chung C, et al. The impact of web-based and face-to-face simulation on patient deterioration and patient safety: Protocol for a multi-site multi-method design. BMC Health Services Res 2016;16(1). https://doi.org/10.1186/s12913-016-1683-0

9. Botma Y, Greef M, Mulaudzi M, et al. Research in Health Sciences. Cape Town: Heinemann Pearson Education, 2010:251-254.

20. Benner P. From Novice to Expert - Excellence and Power in Clinical Nursing Practice. Commemorative Edition. California: Addison-Wesley Publishing Company, 2001:307.

21. Turrill S. The education of UK specialised neonatal nurses: Reviewing the rationale for creating a standard competency framework. Nurse Educ Practice 2014;14(5):504-511. https://doi.org/10.1016/j. nepr.2014.07.001

22. Kenner C, Reinarz S. Interprofessional teamwork and neonatal patient safety: A nursing perspective. Neo Rev 2013;14(10)e480-e482. https://doi.org/10.1542/neo.14-10-e480

23. Pattinson RC. Safety versus accessibility in maternal and perinatal care. S Afr Med J 2015;105(4):261-265. https:// doi.org/10.7196/samj. 9182

24. Spence K. Ethical advocacy based on caring: A model for neonatal and paediatric nurses. J Paediatrics Child Health 2011;47:642-645. https://doi.org/10.1111/j.1440-1754.2011.02178.x

25. Callister L. Culturally competent care of women and newborns: Knowledge, attitude, and skills. J Obstet Gynecol Neonatal Nursing 2006;30(2):209-215. https://doi.org/10.1111/j.1552-6909.2001.tb01537.x

26. World Health Organization. World health statistics 2018. Monitoring health for the SDGs, sustainable development goals. Geneva: WHO, 2018.

27. Esamai F, Lockyer J, Bose C, et al. Evaluation of an educational program for essential newborn care in resourcelimited settings: Essential care for every baby. BMC Pediatrics 2015;15(1):1-11. https://doi.org/10.1186/s12887015-0382-z

28. Burke S. Systematic review of developmental care interventions in the neonatal intensive care unit since 2006. Child Health Care 2018;22(2):269-286. https://doi.org/10.1177/1367493517753085

29. Sharma G, Zaka N, Hailegebriel T. Infection prevention control at neonatal intensive care units. UNICEF, 2018 https://www.healthynewbornnetwork.org/resource/infection-prevention-and-control-at-nicu/ (accessed 8 November 2019)

30. Baley J. Committee on fetus and newborn. Skin-to-skin care for term and preterm infants in the neonatal ICU from the American Academy of Pediatrics: Clinical Report. Pediatrics 2015;136(3):596-599.

31. Kramer MS, Kakuma R. Optimal duration of exclusive breastfeeding. Cochrane Database of Systematic Reviews 2012;8. https://doi.org/10.1002/14651858.CD003517.pub2

32. World Health Organization. Immunization. https://www.who.int/topics/immunization/en/ (accessed 8 November 2019).

33. Gardner SL, Carter BS, Hines ME, et al., (editors). Merenstein and Gardner's Handbook of Neonatal Intensive Care. 8th ed. St. Louis, Missouri: Elsevier, 2016.

34. Verklan MT, Walden M, eds. Core Curriculum for Neonatal Intensive Care Nursing. 5th ed. Pennsylvania: WB Saunders, 2015. 
35. American Academy of Pediatrics. Guidelines and policies. https://www.aap.org/en-us/professional-resources/qualityimprovement/Pages/Guidelines-and-Policy-Development.aspx (accessed 8 November 2019).

(3) 2019).

(accessed 8 November 2019)

38. Ohning BL, Rosenkrantz T. Transport of the critically ill newborn. Pediatrics: Cardiac disease and critical care medicine. Medscape, 2019. https://emedicine.medscape.com/article/978606-overview\#showall (accessed 8 November 2019).

39. Te Pas AB. Improving neonatal care with technology. Front Pediatr 2017;5:110. https://do org/10.3389\%2Ffped.2017.00110

40. Limpopo Initiative for Newborn Care. Norms and standards for essential newborn care, 2016. http://www lincare.co.za/wp-content/uploads/2016/06/Chapter-2-Norms-and-Standards-for-Essential-Newborn-Care.pd (accessed 10 November 2018).

41. Vetcho S, Cooke M, Ullman A. Family-centred care in dedicated neonatal units: An integrative review of international perspectives. J Neonatal Nurs 2020;26(2):73-92. https://doi.org/10.1016/j.jnn.2019.09.004

2. Pattinson RC, Rhoda N. 2012 - 2013: Ninth report on perinatal care in South Africa. Saving Babies. Pretoria Thhepesa Press, 2014:35 http://www.ppip.co.za/wp-content/uploads/SavingBabies-2012-2013.pdf (accessed 10 NovemTshepesa P.
ber 2018).

43. Montanholi LL, Merighi MAB, de Jesus MCP. The role of the nurse in the neonatal intensive care unit: Between the ideal, the real and the possible. www.eerp.usp.br/rlae 2011:307 (accessed 6 July 2020).

44. Petty J. A global view of competency in neonatal care. J Neonatal Nurs 2014;20:3-10. https://doi.org/10.1016/ nnn.2013.05.001
45. Melnyk BM. Editorial: The difference between what is known and what is done is lethal: Evidence-based practice is a key solution urgently needed. Worldviews Evidence-Based Nurs: Sigma Theta Tau Internation 2017;14(1):3-4. https://doi.org/10.111/wvn.12194

6. South Africa. Nursing Act No. 33 of 2005. Regulations 786: The scope of practice of nurses and midwives. Pretoria: Government Printers, 2013

47. Oestergaard MZ, Inoue M, Yoshida S, et al. Neonatal mortality levels for 193 countries in 2009 with trend since 1990: A systematic analysis of progress, projections and priorities. PLoS Med 2011. https://doi.org/10.1371/ journal.pmed.1001080 (accessed 14 November 2019).

48. Eklund W, Kenner C. Council of International Neonatal Nurses - Global Neonatal Provider Database Initiative Results from an implementation focus group. Adv Neonatal Care 2015;15(6):407-415. https://doi.org/10.1097/ ANC. 0000000000000236

49. Half R. Professional development training: A win for the entire team. 2017. https://www.roberthalf.com/blog management-tips/professional-development-training-a-win-for-the-entire-team (accessed 3 March 2020).

50. United Nations. Sustainable Development Knowledge Platform. New York: UN, 2015. https: sustainabledevelopment.un.org/partnerships/unsummit2015 (accessed 3 March 2020).

Accepted 23 July 2020. 